\title{
The Determinant of Potyvirus Ability to Overcome the RTM Resistance of Arabidopsis thaliana Maps to the N-Terminal Region of the Coat Protein
}

\author{
V. Decroocq, ${ }^{1}$ B. Salvador, ${ }^{2}$ O. Sicard, ${ }^{1}$ M. Glasa, ${ }^{3}$ P. Cosson, ${ }^{1}$ L. Svanella-Dumas, ${ }^{1}$ F. Revers, ${ }^{1}$ \\ J. A. García, ${ }^{2}$ and T. Candresse ${ }^{1}$ \\ 'UMR GDPP, INRA Université Bordeaux II, IBVM, Centre INRA de Bordeaux, BP 81, 33883 Villenave d'Ornon Cedex; \\ France; ${ }^{2}$ Centro Nacional de Biotecnología (CSIC), Campus de la Universidad Autónoma de Madrid, 28049 Madrid, Spain; \\ ${ }^{3}$ Institute of Virology, Department of Plant Virology, Slovak Academy of Sciences, Dúbravská cesta 9, 84505 Bratislava, \\ Slovakia
}

Submitted 9 March 2009. Accepted 28 May 2009.

In Arabidopsis thaliana Columbia (Col-0) plants, the restriction of Tobacco etch virus (TEV) long-distance movement involves at least three dominant RTM (restricted TEV movement) genes named $R T M 1, R T M 2$, and $R T M 3$. Previous work has established that, while the RTM-mediated resistance is also effective against other potyviruses, such as Plum pox virus (PPV) and Lettuce mosaic virus (LMV), some isolates of these viruses are able to overcome the RTM mechanism. In order to identify the viral determinant of this RTM-resistance breaking, the biological properties of recombinants between PPV-R, which systemically infects Col-0, and PPV-PSes, restricted by the RTM resistance, were evaluated. Recombinants that contain the PPV$R$ coat protein (CP) sequence in an RTM-restricted background are able to systemically infect Col-0. The use of recombinants carrying chimeric $\mathrm{CP}$ genes indicated that one or more PPV resistance-breaking determinants map to the $5^{\prime}$ half of the CP gene. In the case of $\mathrm{LMV}$, sequencing of independent RTM-breaking variants recovered after serial passages of the LMV AF199 isolate on Col-0 plants revealed, in each case, amino acid changes in the $C P$ N-terminal region, close to the DAG motif. Taken together, these findings demonstrate that the potyvirus CP N-terminal region determines the outcome of the interaction with the RTM-mediated resistance.

Plant viruses are systemic pathogens that infect a susceptible host in a multistep process. Consecutive to entry in host cells, replication of the viral genome is followed by virus movement to neighboring cells through the plasmodesmata (also called cell-to-cell movement), presumably utilizing the cellular pathways of plasmodesmal transport (Waigmann et al. 2004), and over longer distances through the plant vascular bundles (the long-distance movement), leading to systemic infection (Gilbertson and Lucas 1996; Wang et al. 1999). Longdistance movement occurs through the phloem for most viruses, following the photoassimilate path from source to sink organs. Thus, in order to successfully infect distant tissues, viruses must enter the vascular system, access, move through, and exit from

Corresponding author: V. Decroocq; E-mail: decroocq @bordeaux.inra.fr

* The $\boldsymbol{e}$-Xtra logo stands for "electronic extra" and indicates that three supplemental tables are published online. sieve tubes, exit the vascular system, and infect nonvascular cells of sink tissues. Depending on the virus, viral genomic nucleic acids are transported as either virions or nucleoprotein complexes (Gilbertson and Lucas 1996).

Our understanding of the mechanisms controlling long-distance movement remains limited, and the role of phloem sap proteins in long-distance movement of virus particles has yet to be defined. It has been suggested that plant viruses are passively transported along with the solutes through the phloem or that they undergo selective unloading at specific cell boundaries (Lucas 2006). These last observations support the notion that the long-distance movement of viruses in the sieve-tube system may not simply follow the stream of assimilates and that phloem exit in sink tissues is highly controlled. Moreover, viral destination appears to be regulated by protein-protein or protein-RNA interactions, or both, within phloem tissues (Kehr and Buhtz 2008; Lough and Lucas 2006). Numerous viral determinants involved in potyvirus infection were identified, among which three potyviral proteins seem to be involved in long-distance movement, e.g., the coat protein (CP), the helper component-proteinase (HC-Pro), and the genome-linked protein (VPg) (Revers et al. 1999). Conversely, nearly nothing is known about host factors involved in the long-distance process of potyviruses.

Genetic and functional genomic studies in Arabidopsis have revealed the importance of specific host proteins in controlling the vascular movement of Tobacco etch virus (TEV) (Whitham et al. 1999). During infection with this potyvirus, at least three RTM (restricted TEV movement) genes, most probably acting in synergy, prevent systemic but not local TEV infection in Col-0 and WS wild-type plants (Mahajan et al. 1998). In comparison, some other Arabidopsis accessions, i.e., Ler (Landsberg erecta) and C24, allow TEV long-distance movement from inoculated rosette leaves to noninoculated inflorescence tissues. The RTM1 and RTM2 genes have been identified by map-based cloning (Chisholm et al. 2000; Whitham et al. 2000). RTMI encodes a jacalin-like lectin protein, while RTM2 is related to the multigene family of small heat-shock proteins. The RTM1 and RTM2 proteins are expressed mostly or exclusively in the phloem (Chisholm et al. 2001). In fact, these proteins contribute to a dominant blockage of systemic infection of Col-0 plants by at least three distinct potyviruses, namely, TEV, Plum pox virus (PPV), and Lettuce mosaic virus (LMV) (Decroocq et al. 2006; Mahajan et al. 1998). The three controlled potyviruses are able to establish a systemic infec- 
tion in Col-0 plants only when at least one of the RTM genes is mutated (Decroocq et al. 2006; Mahajan et al. 1998). One fascinating challenge with the RTM resistance is the understanding of the mechanism or mechanisms by which the RTM gene products operate. One way to address this issue is to gain knowledge on one or more viral determinants interacting with the RTM factors.

We have previously reported that, whereas the long distance movement of PPV isolates is restricted by the RTM resistance, one PPV isolate, PPV-Rankovic (PPV-R), is able to overcome the RTM resistance in the Arabidopsis Col-0 accession (Decroocq et al. 2006). In the present work, the use of a reverse genetics approach involving the construction of recombinants between PPV-R (RTM-breaking isolate) and PPV-PS (RTM-restricted isolate) allowed the mapping of one or more PPV RTM-breaking determinants to the $\mathrm{N}$-terminal region of the viral CP. This result is further confirmed by the analysis of the biological properties of recombinants involving other PPV isolates as parents and by the sequencing of RTM-breaking variants selected for another potyvirus controlled by the RTM system, LMV. In the case of LMV, the overcoming of the RTM resistance was found to be associated with point mutations affecting amino acids of the LMV CP N-terminal region. These results are further discussed in terms of the manner in which the RTM complex and viral CP might interplay to restrict the virus phloem long distance transport.

\section{RESULTS}

\section{Restriction of systemic infection of some PPV isolates in the Col-0 ecotype of Arabidopsis is caused by the RTM resistance.}

We previously showed that, while the PPV-EA (PPV-ElAmar) long-distance movement is restricted in the Col-0 accession, several other PPV isolates including PPV-R and PPVNAT (non-aphid transmissible) (two members of the PPV-D strain) are able to invade and accumulate in noninoculated tissues (Decroocq et al. 2006). To examine the ability of other PPV isolates or strains to systemically invade Col-0, the accumulation of PPV-PSes and PPV-SK68 (both belonging to the PPV-M strain) was scored by enzyme-linked immunosorbent assay (ELISA) at 9, 15, 21, and 28 days postinoculation (dpi) in mechanically inoculated and noninoculated Col-0 tissues. The previously tested PPV-EA and PPV-R were used as controls. Failure to accumulate, as judged by ELISA, was confirmed by reverse transcription-polymerase chain reaction (RTPCR) analysis.

All tested PPV isolates were detected in inoculated leaves at 9 and $15 \mathrm{dpi}$. In noninoculated inflorescence tissues (leaves and flowers), PPV-Pses, and PPV-SK68, the two isolates of the PPV-M strain behaved as PPV-EA and could not be detected at any timepoint. On the contrary, accumulation of PPV-NAT and PPV-R, both belonging to the PPV-D strain, was readily detected at $21 \mathrm{dpi}$ in noninoculated tissues (Supplementary Table S2). The inability of PPV-PSes and PPV-SK68 isolates to establish a systemic infection was confirmed even upon direct biolistic inoculation of Col-0 plants, and similar results were again obtained using the green fluorescent protein (GFP)tagged version of PPV-PSes. Infectivity of the PPV-PSes and PPV-SK68 cDNA clones was determined, in parallel, by direct biolistic inoculation of Ler plants and of rtm mutants (data not shown), which bear point-mutated, homozygous alleles of either the RTM1, RTM2, or RTM3 genes in a Col-0 background (Whitham et al. 1999). All Arabidopsis Ler and rtm plants inoculated with PPV-PSes and PPV-SK68 were tested positive for infection of both inoculated and noninoculated leaves at 9 and $21 \mathrm{dpi}$, respectively.
Overall, the results obtained demonstrate that similar to PPVEA, the PPV-PSes and PPV-SK68 isolates are unable to systemically invade the Col-0 Arabidopsis accession, because their long-distance movement is restricted by the action of the RTM resistance system. On the other hand, the ability of both PPVNAT and PPV-R to systemically invade Col-0 indicates that they are RTM-breaking isolates, opening the way to the identification of the region of the viral genome contributing this property, using chimeras between the PPV-R and PPV-PSes genomes.

The GFP-tagged version of PPV-R (pICPPVnk-GFP) displays properties similar to those of its untagged parental isolate. Figure 1 shows that PPVnk-GFP, progeny of the inoculated pICPPVnk-GFP, is able to establish a systemic infection in the wild-type Col-0 by $21 \mathrm{dpi}$, thereby breaking the RTM resistance (Fig. 1A and D). On the contrary, the P/R(GFP) 7677 8576 viral clone, corresponding to the PPV-PSes isolate that contains one single 101-bp PPV-R fragment from position 7677 to 8576 (Fig. 2) is restricted to the initially inoculated Col-0 leaves. Its behavior is similar to that of the PPV-PSes isolate, it can move from cell to cell (Fig. 1B and 1C) but it cannot infect inflorescence tissues (Fig. 1E). Besides, a single mutation in either the RTM1, RTM2, or RTM3 genes is sufficient to alleviate the restriction of $\mathrm{P} / \mathrm{R}(\mathrm{GFP}) 7677-8576$ longdistance movement and systemic infection of Col-0 plants, since GFP expression was detected at $21 \mathrm{dpi}$ in upper noninoculated leaves of the three mutants (Fig. 1G, H, and I).

\section{The PPV-R-derived RTM-breaking determinants map in the $5^{\prime}$ region of the $C P$ gene.}

Because of the differential behavior of PPV-PSes and PPV-R towards the RTM resistance, the RTM-breaking properties of seven available recombinant constructs between these two isolates, including the two GFP-tagged ones, P/R(GFP) 7677 8576 and P/R(GFP) 7677-9020, were initially assessed (Fig. 2). Six to twelve Col-0 plants were thus mechanically inoculated, using recombinant virus isolates first propagated in Nicotiana benthamiana. Infection of Col-0 plants was monitored at $9 \mathrm{dpi}$ in inoculated leaves and at $21 \mathrm{dpi}$ in noninoculated inflorescence tissues (leaves and flower buds) by fluorescence for P/R(GFP) 7677-9020 and by ELISA and RT-PCR for the other five recombinants. The experiment was repeated three times, with similar results. Although not all symmetrical recombinant pairs are available, the results with the five recombinants that are not GFP-tagged are consistent with a role of the $3^{\prime}$ region of the genome, corresponding to half of the $\mathrm{NIb}$ gene, the $\mathrm{CP}$ gene, and the $3^{\prime}$ noncoding region. This is indicated in particular by the fact that PPV R/P 7677 is not able to systemically infect Col-0 inflorescences while PPV R/P 2212-7677 develops a systemic infection in this host.

In addition, while P/R(GFP) 7677-8576 long-distance movement is restricted in Col-0 plants (Figs. 1E and 2), the P/R(GFP) 7677-9020 construct is able to infect Col-0 inflorescences (Figs. 1F and 2). The only difference between these two PPVPS constructs is the presence in P/R(GFP) 7677-9020 of the 5' half of the CP gene (from the first nucleotide to the SacI restriction site) of the PPV-R isolate, which suggests that the CP or, more precisely, the first 146 amino acids of the $\mathrm{N}$-terminal $\mathrm{CP}$ region is the viral factor involved in the overcoming of the RTM resistance by PPV-R.

As a control that the restriction of the systemic movement of the recombinants was indeed caused by the RTM resistance, all recombinants were also inoculated on rosettes of the Ler accession and of the various rtm mutants. Viral infection was assessed by ELISA or, if possible, GFP observation in the uninoculated upper leaves at $21 \mathrm{dpi}$. As expected, all recombinants, irrespective of their behavior in Col-0, were readily detected (data not shown) in uninoculated upper leaves of Ler 
plants or of the various rtm mutants. The identity of the various PS/R recombinants used in those experiments was verified by partial resequencing of the PPV genome in regions encompassing the recombination sites. These control experiments demonstrate that all tested PPV constructs are fully infectious in Arabidopsis and that the impaired systemic accumulation observed in Col-0 for some of them is not due to a nonspecific loss of infectivity but, rather, to the RTM-mediated resistance mechanism active in this host.

\section{The first 146 amino acids of the CP control the behavior} of PPV isolates towards the RTM resistance.

To experimentally validate the contribution of the first 146 amino acids of the $\mathrm{CP}$ to the resistance phenotype in Col- 0 , a series of PPV recombinant cDNA clones was designed to exchange this $\mathrm{N}$-terminal $\mathrm{CP}$ region between various PPV isolates. This region was symmetrically exchanged between PPV-R and PPV-PSes, yielding the R/P 8576-9020 and P/R 8576-9020 recombinant pair. In addition, the N-terminal region of PPV-EA was introduced in PPV-R and that of PPV-SK68 in PPV-PSes, yielding recombinants R/EA 8576-9020 and P/SK68 8576-9020, respectively.

The results obtained show that replacing the sequence coding for N-terminal CP region of PPV-R by a similar KpnI/SacI fragment issued from PPV-PSes or PPV-EA compromises virus systemic accumulation in Col-0 plants (Fig. 2) but not in Ler or rtm mutants (data not shown). Conversely, replacing the PPV-PSes N-terminal CP region by that of PPV-R (but not that
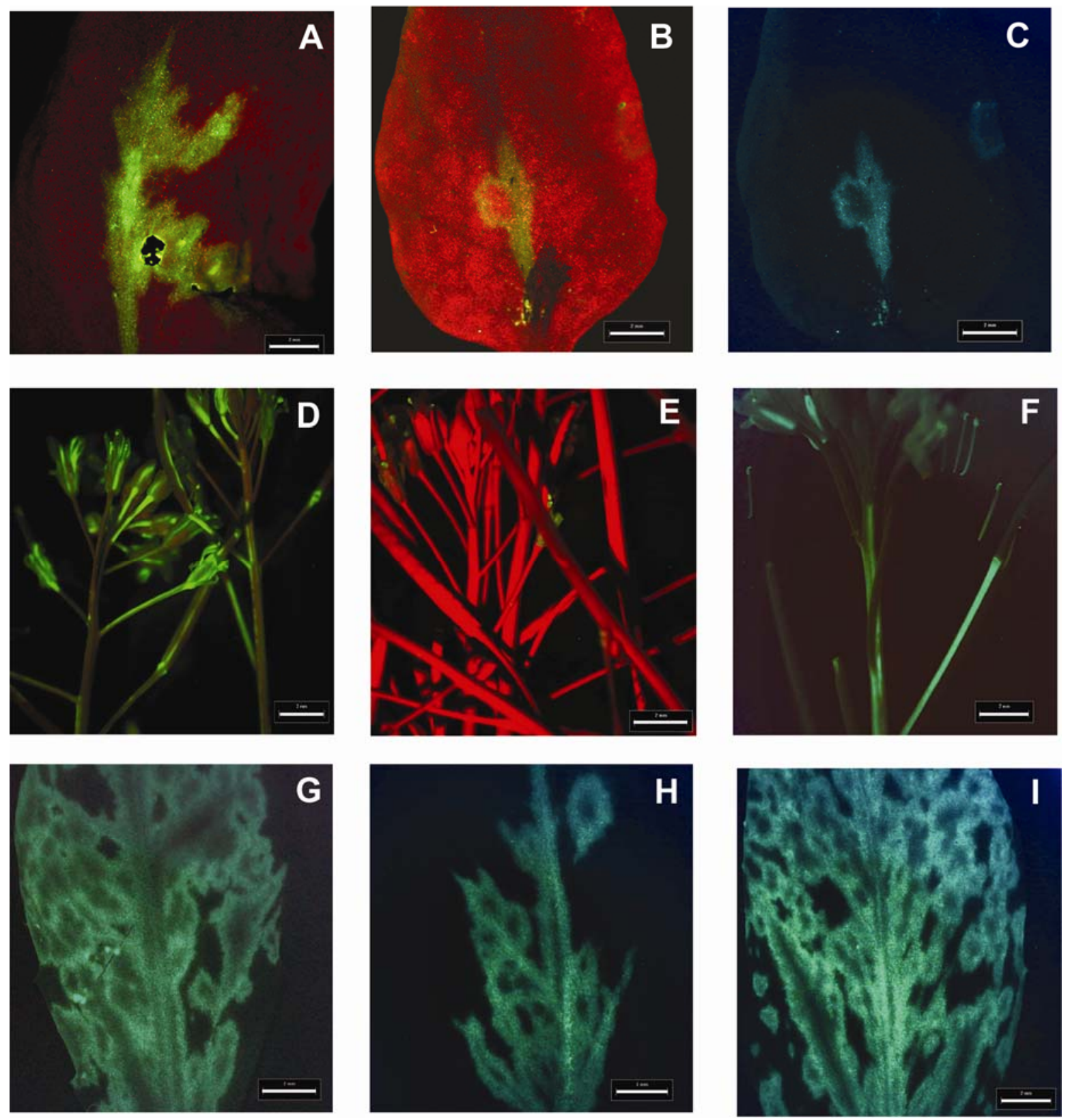

Fig. 1. Distribution of green fluorescent protein (GFP)-tagged Plum pox virus (PPV) isolates within inoculated rosette leaves and uninoculated inflorescences of Col-0 wild-type or rtm-mutated plants. Photographs were taken under UV light as described by Decroocq and associates (2006). A, Inoculated rosette leaves at 9 days postinoculation (dpi) and $\mathbf{D}$, uninoculated inflorescence tissues at 21 dpi of Col-0 plants infected with the restricted TEV movement (RTM)breaking isolate, pICPPVnk-GFP (PPV-R). B, Stereomicroscopy images obtained with a B or C, a GFP3 filter at 9 dpi from a rosette leaf of a Col-0 plant inoculated with P/R(GFP) 7677-8576; E, Images obtained at 21 dpi from uninoculated inflorescences of Col-0 plants infected with P/R(GFP) 7677-8576 or F, P/R(GFP) 7677-9020. G through I, Uninoculated inflorescence and rosette leaves of Col-0 mutants rtm1-1, rtm1-2, and rtm3-1, respectively, inoculated with P/R(GFP) 7677-8576. Bar $=2 \mathrm{~mm}$. 
of PPV-SK68, a non-resistance breaking isolate) resulted in a recombinant virus that systemically accumulated in Col-0 plants (Fig. 2), demonstrating that the exchanged CP N-terminal region is sufficient to determine the systemic invasion phenotype in Col-0 plants.

\section{Amino acid changes in the $\mathrm{N}$-terminal region of the LMV CP correlate with the ability to overcome the RTM resistance.}

While the LMV-AF199 isolate is able to systemically infect numerous Arabidopsis thaliana accessions, it is restricted to the inoculated leaves in Col-0 (Revers et al. 2003) and this long-distance movement-restriction phenotype has been shown to be mediated by the RTM resistance (Decroocq et al. 2006). However, systemic accumulation of LMV-AF199 in Col-0 plants is occasionally observed in a low percentage of the inoculated plants (not shown). Serial propagation in Col-0 of the viral isolate present in these plants results in the selection of LMV-AF199 variants able to systemically infect $100 \%$ of the inoculated Col-0 plants, as revealed by ELISA and RTPCR assays (data not shown). In this way, a total of four RTMbreaking variants were independently obtained, deriving from independent inoculation experiments of Col-0 plants. Given the involvement of the PPV CP N-terminal region in RTMbreaking, initial characterization was carried out on one of these variants, LMV-AFVAR1, by sequencing its $\mathrm{N}$-terminal $\mathrm{CP}$ region and comparing it with that of the original LMVAF199 inoculum maintained in lettuce. A single G to A mutation at position 9,063 of the genome causing a glycine to aspartic acid amino acid change at position 10 of the $\mathrm{CP}$ was identified (Fig. 3). Because no infectious cDNA clone of LMV-AF199 is available, site-directed mutagenesis cannot be used to validate the effect of this mutation on the phenotype towards the RTM resistance. As an alternative the full genome sequence of LMV-AFVAR1 was determined and compared with its parent LMV-AF199, and three other independent RTM-breaking variants were characterized.

In total, two additional mutations were identified between the complete genome of LMV-AFVAR1 and that of its LMVAF199 parent, a $G$ to A mutation at position 3,883 of the genome in the $\mathrm{P} 3$ coding region and a $\mathrm{C}$ to $\mathrm{T}$ mutation at position 8,729 within the NIb coding region. However, these two mutations are synonymous ones, which do not change the sequence of the viral polyprotein, so that LMV-AFVAR1 differs by a single amino acid change in the $\mathrm{CP} \mathrm{N}$-terminal region from its LMV-AF199 progenitor (Fig. 3).

Analysis of the three other independently obtained RTMbreaking variants was carried out by partial sequencing in the regions surrounding the mutations identified in LMV-AFVAR1. None of these three variants contained the silent mutations at

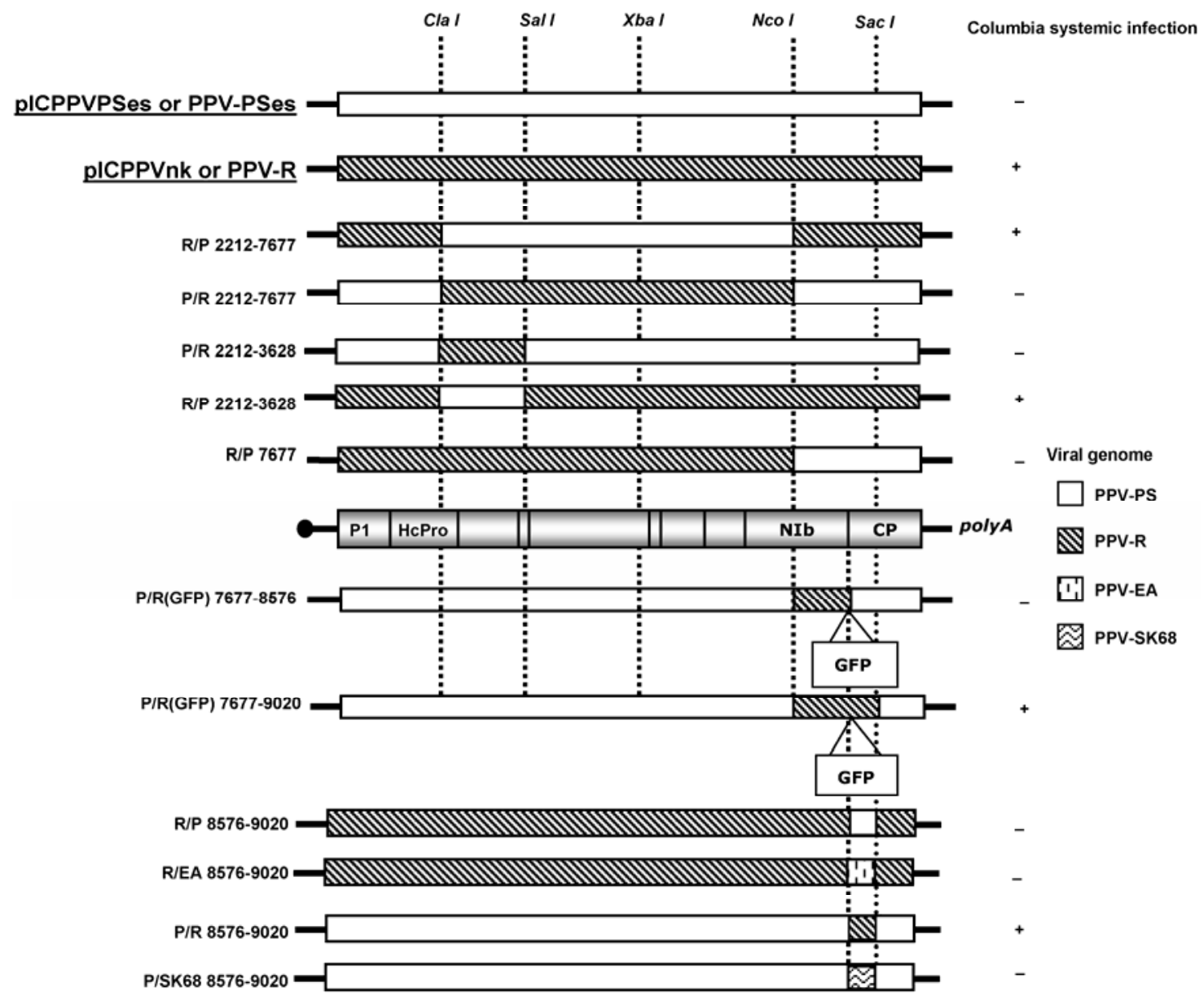

Fig. 2. Schematic representation of the Plum pox virus (PPV) genome and of different PPV recombinants tested on the Col-0 accession. The outcome of Col0 infection with PPV isolates and recombinants is depicted on the right: + indicates systemic infection at 21 dpi was detected and - that no systemic infection of uninoculated inflorescences detected at 21 dpi. PPV-PSes and PPV-R sequences are shown as open and filled boxes, respectively. Restriction sites used in the cloning are indicated on the top. A genetic map of PPV, indicating the positions of the encoded proteins, is shown in the middle. The names of the virus proteins are indicated in the PPV genome map as detailed by Urcuqui-Inchima and associates (2001). Infectious clones and recombinants are fully described in this manuscript and by Sáenz and associates (2000). The location of the introduced green fluorescent protein (GFP) open reading frame is indicated underneath the corresponding PPV construct. 
positions 3,883 or 8,729 (result not shown), but all three of them contained nonsynonymous mutations in their CP N-terminal region (Fig. 3). One variant presents the same mutation as LMV-AFVAR1 at position 10 of the $\mathrm{CP}$, while the other two show, respectively, a serine to asparagine mutation at position 11 (LMV-AFVAR2) or an aspartate to glycine mutation at position 14 of the CP (LMV-AFVAR3) (Fig. 3).

\section{Biophysical parameters of the $\mathrm{N}$-terminal $\mathrm{CP}$ region in PPV and LMV.}

Previous work has demonstrated the influence of CP phosphorylation, $O$-glycosylation, and $\mathrm{N}$-terminal net charge on potyvirus infectivity or ability to mount systemic infection in host plants (Chen et al. 2005; Fernández- Fernández et al. 2002; Ivanov et al. 2003). In order to evaluate such a possibility in the case of the RTM resistance, the isoelectric point, net charge, and total number of predicted potential sites for phosphorylation or glycosylation ( $\mathrm{Thr}+$ Ser residues) were compared for the PPV and LMV CP N-terminal regions of RTM-restricted and RTM-breaking isolates (Table 1).

These computations show that the original wild-type LMV $\mathrm{CP}$ and one of its variants (LMV-AFVAR1) contain the same number of positively and negatively charged amino acids, giv- ing an equivalent slightly negative net charge (Table 1). Surprisingly, in LMV-AFVAR3, the terminal CP net charge is null, which is commonly found to be deleterious to potyvirus systemic movement in host plants like zucchini squash, tobacco, and N. benthamiana (Kimalov et al. 2004; López-Moya and Pirone 1998). In comparison, the N-terminal CP net charge of all PPV isolates is significantly negative. However, multiple amino acid changes between PPV-NAT (an RTM breaking isolate) and PPV-SK68 (an RTM-restricted isolate) did not modify the total net charge $(-6)$ of this CP region. Overall, this analysis does not reveal a clear correlation between the CP Nterminal net charge and the ability to overcome the RTM resistance in Col-0. Interestingly, while the LMV wild-type isolate and two of its variants display similar amounts of Thr and Ser residues, the PPV RTM-restricted isolates (PPV-PSes, PPV-SK68, PPV-EA) harbor a significantly higher number of Thr residues (Table 1) in comparison to the PPV RTMbreaking isolates (PPV-R and PPV-NAT). However, the situation is reversed concerning the Ser phosphorylation or glycosylation sites. Moreover, an RTM-breaking isolate, PPV-R, and the RTM-restricted isolate PPV-SK68 display a similar proportion of $\mathrm{Thr}+$ Ser residues $(20 \%)$. Therefore, we cannot conclude on a significant correlation between the number of

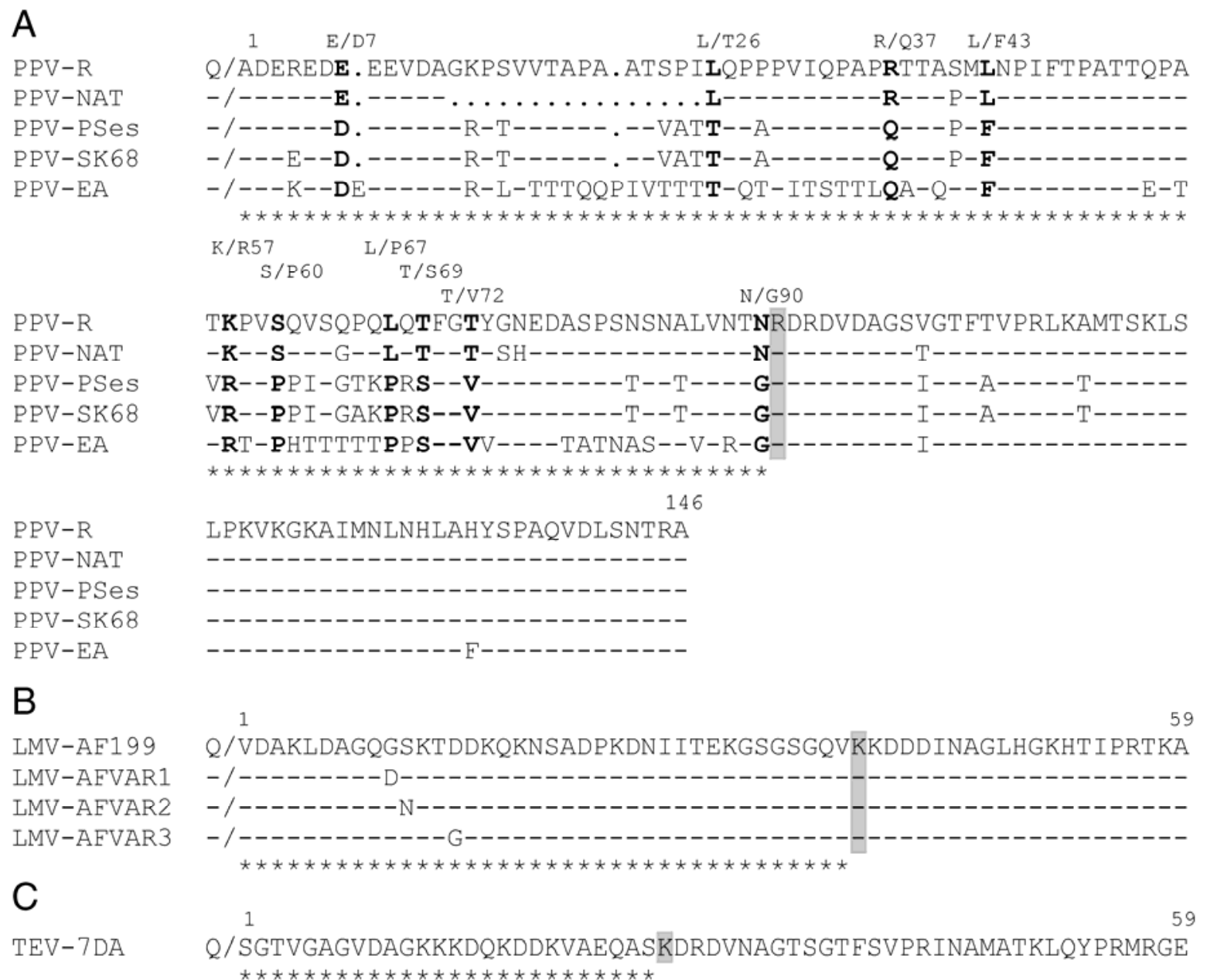

Fig. 3. Alignment of the amino acid sequences of A, Plum pox virus (PPV), B, Lettuce mosaic virus (LMV), and C, Tobacco etch virus (TEV) N-terminal coat protein $(\mathrm{CP})$ regions. Identical amino acids are indicated by horizontal bars. The 15 amino acid 'NAT' deletion is represented by dots. The amino acid highly variable N-terminal sequence used for the analysis of the CP biophysical parameters is underlined with stars. The amino-acids highlighted in gray correspond to the start of the core conserved region of PPV, LMV and TEV CP. Residues in bold are those consistently differing between restricted TEV movement (RTM)-breaking (PPV-R and PPV-NAT) and RTM-restricted (PPV-PSes, PPV-SK68, and PPV-EA) isolates. The corresponding amino acid substitutions are represented above the PPV sequence. The sequences of PPV-R and PPV-PSes used in this alignment correspond to the CP sequences of pICPPVnk and pICPPVPSes isolates, respectively. The TEV-7DA (named TEV-GUS) N-terminal CP sequence depicted in this figure belongs to the TEV isolate initially used to identify host genes controlling RTM in Arabidopsis (Mahajan et al. 1998; Whitham et al. 1999). 
potentially phosphorylated or glycosylated residues and RTM resistance.

\section{Systemic infection of RTM-restricted and RTM-breaking PPV and LMV isolates in the $O G T$ mutant plants.}

To examine the ability of RTM-restricted PPV and LMV isolates to overcome the RTM resistance in $O G T$ ( $O$-linked $\mathrm{N}$-acetylglucosamine transferase)-depleted mutant plants, the accumulation of PPV-PSes, PPV-EA, and LMV-AF199 was scored by ELISA at 9 and $21 \mathrm{dpi}$, in mechanically inoculated and noninoculated tissues of Arabidopsis knock-out mutants (Supplementary Table S3). The previously tested PPV-R (pICPPVnkGFP; Chen et al. 2005) was used as control. In comparison, the OGT Col-0 mutants (namely, sec-2 and spy-1) were also inoculated with the RTM-breaking LMV variants (LMV-AFVAR 1 to LMV-AFVAR 3). Failure to accumulate, as judged by ELISA, was confirmed by RT-PCR analysis. In this study, only the RTM-breaking PPV isolate and LMV-AFVAR variants were able to complete a successful systemic infection in the $O G T$-mutant plants. In consequence, reduction of the activity of one or the other Arabidopsis OGT did not alleviate the RTM resistance.

\section{DISCUSSION}

In Arabidopsis, the RTM-mediated restriction of potyvirus long-distance movement involves at least three host proteins, i.e., RTM1, RTM2, and RTM3 (Chisholm et al. 2001; Mahajan et al. 1998; Whitham et al. 1999). This resistance machinery is active against at least three distinct potyviruses, TEV, PPV, and LMV (Decroocq et al. 2006; Mahajan et al. 1998). Since all TEV isolates initially tested presented the same restriction phenotype, no information about one or more viral factors interacting with the RTM proteins, or at least relevant for the RTM resistance, could be obtained. We previously reported that several PPV isolates, PPV-R among them, are able to overcome the RTM resistance in the Arabidopsis Col-0 accession (Decroocq et al. 2006). In the present study, we extend the number of RTM-breaking and RTM-restricted PPV isolates. Here, we show clearly that, similarly to TEV (Mahajan et al. 1998), the RTM-restricted PPV-PSes isolate is not defective in accumulation and cell-to-cell movement within the inoculated leaf but is, rather, deficient in establishing an effective systemic infection of Col-0 plants (Fig. 1). The two identified RTMbreaking isolates belong to the PPV-D strain, while the RTMrestricted isolates belong either to the PPV-M or PPV-EA strains. The first PPV-PS isolate, initially tested in Col-0
(Decroocq et al. 2006; Nicaise et al. 2007) and which appeared to break down the RTM resistance, was later on resequenced and shown to belong to the PPV-D strain and to be a close variant of PPV-R (V. Decroocq, unpublished data). Whether the behavior towards the RTM resistance is a homogenous property shared by all isolates within a given PPV strain will have to be specifically investigated.

\section{The sequence of the $\mathrm{N}$-terminal region of the $\mathrm{CP}$ determines the breaking of the RTM-mediated resistance in Col-0 plants.}

A reverse-genetics approach was used to map the viral determinants of RTM-breaking by analyzing the infection phenotype in Col-0 plants of recombinant constructs between PPV-R and PPV-PSes (Fig. 2). This allowed the identification of a key role for the region encoding the first 146 amino acids of the CP. However, PPV RTM-breaking and RTM-restricted isolates are substantially divergent within their $\mathrm{N}$-terminal $\mathrm{CP}$ region (Fig. 3), making it difficult to pinpoint single amino acids that could be relevant for RTM-resistance breaking.

In the case of LMV, complete genome comparison between RTM-breaking variants and the non-resistance breaking progenitor allowed the identification of three-point mutations, a single one of which changes the sequence of the viral polyprotein. Although a contribution of the two noncoding mutations to the resistance-breaking phenotype cannot be absolutely ruled out, the fact that none of these mutations was observed in the three other independently obtained resistance-breaking variants strongly argues against such a hypothesis. On the contrary, the fact that the glycine to aspartate mutation at position 10 of the $\mathrm{CP}$ was observed in two independently obtained variants is a very strong indication of its implication in the resistance-breaking phenotype. This is further supported by the observation of coding mutations at closely located positions of the CP of two other independently obtained resistance-breaking variants (Fig. 3). Interestingly, despite the relative ease with which resistance-breaking variants could be selected for LMV, it was not possible to select similar variants for PPV, despite extensive efforts involving the inoculation of batches of 24 to 48 Col-0 plants with three different PPV isolates (pICPPVPSes, PPV-SK68, or PPV-EA).

\section{Different models for the RTM-mediated restriction of viral long-distance movement.}

The CP of plant viruses is multifunctional (Urcuqui-Inchima et al. 2001). In addition to its obvious implication in the encap-

Table 1. Biophysical parameters characterizing Plum pox virus (PPV), Lettuce mosaic virus (LMV), and Tobacco etch virus (TEV) N-terminal coat protein (CP) regions ${ }^{\mathrm{a}}$

\begin{tabular}{|c|c|c|c|c|c|c|c|}
\hline Viral CP & $\begin{array}{l}\mathrm{N} \text {-terminal } \\
\text { region }\end{array}$ & pI & $\begin{array}{l}\text { NIb negatively } \\
\text { charged residues }\end{array}$ & $\begin{array}{l}\text { NIb positively } \\
\text { charged residues }\end{array}$ & Net charge $^{d}$ & $\begin{array}{c}\text { \% Target residues for } \\
\text { phosphorylation }\end{array}$ & $\begin{array}{c}\text { RTM- } \\
\text { breaking }\end{array}$ \\
\hline PPV-R & AD...NTN & 4.21 & 9 & 4 & -5 & $12.2 \%(\mathrm{~T}) 7.8 \%(\mathrm{~S})$ & Yes \\
\hline PPV-NAT & AD ...NTN & 4.20 & 9 & 3 & -6 & $11.8 \%(\mathrm{~T}) 7.9 \%(\mathrm{~S})$ & Yes \\
\hline PPV-PSes & $\mathrm{AD} \ldots \mathrm{VNT}$ & 4.36 & 9 & 5 & -4 & $15.6 \%(\mathrm{~T}) 5.6 \%(\mathrm{~S})$ & No \\
\hline PPV-SK68 & $\mathrm{AD} \ldots \mathrm{VNT}$ & 4.11 & 10 & 4 & -6 & $14.4 \%(\mathrm{~T}) 5.6 \%(\mathrm{~S})$ & No \\
\hline PPV-EA & AD...RTG & 4.16 & 12 & 4 & -8 & $27.7 \%(\mathrm{~T}) 3.2 \%(\mathrm{~S})$ & No \\
\hline LMV-AF199 & VD...GQV & 4.92 & 7 & 6 & -1 & $5.3 \%(\mathrm{~T}) 10.5 \%(\mathrm{~S})$ & No \\
\hline LMV-AFVAR1 & VD...GQV & 4.92 & 7 & 6 & -1 & $5.3 \%(\mathrm{~T}) 7.9 \%(\mathrm{~S})$ & Yes \\
\hline LMV-AFVAR2 & VD...GQV & 4.61 & 8 & 6 & -2 & $5.3 \%(\mathrm{~T}) 10.5 \%(\mathrm{~S})$ & Yes \\
\hline LMV-AFVAR3 & VD...GQV & 6.20 & 6 & 6 & 0 & $5.3 \%(\mathrm{~T}) 10.5 \%(\mathrm{~S})$ & Yes \\
\hline TEV-7DA & SG...QAS & 5.92 & 5 & 5 & 0 & $3.8 \%(\mathrm{~T}) 7.7 \%(\mathrm{~S})$ & No \\
\hline
\end{tabular}

a The predicted isolelectric point (pI) and the net charge were determined from the nonconserved amino-acid region of the PPV and LMV CP, using the ExPASy ProtParam tool.

b Total number of negatively charged residues (Asp + Glu).

c Total number of positively charged residues (Arg + Lys).

${ }^{\mathrm{d}}$ Net charge calculated as follows: (Arg + Lys) - (Asp + Glu). 
sidation of the genomic RNA, the potyviral $\mathrm{CP}$ has been shown to play roles in vector transmission (Atreya et al. 1990; Blanc et al. 1997), plasmodesmatal gating (Rojas et al. 1997), and cell-to-cell (Dolja et al. 1995) and long distance (Andersen and Johansen 1998) movement, and the CP of one potyvirus has been shown to display NTPase activity (Rakitina et al. 2005). In PPV, the N-terminal region of the CP was linked to aphid transmission (Maiss et al. 1989) and to virus spread and accumulation (Chen et al. 2005). Dolja and associates (1995) have shown that the $\mathrm{N}$ - and $\mathrm{C}$ - terminal regions of TEV CP are necessary for long-distance movement. The $\mathrm{N}$-terminal region of the CP is broadly variable in length and sequence between potyviruses and, at a lower level, within potyvirus species, so that its sequence is frequently used to distinguish strains within the same virus species (Shukla and Ward 1989). Various authors have proposed that this variability could represent a way for the virus to modulate its interaction with specific host factors involved in cell-to-cell or long-distance movement. In this concept, amino acid variation presumably modifies the ability of the virus to interact with one or more host components of a movement complex and, in consequence, alters the capacity of the virus for systemic movement (López-Moya and Pirone 1998). The first hypothesis concerning the mechanism underlying the RTM resistance is that the N-terminal region of $\mathrm{CP}$ interacts with host factors to potentiate virus movement and that the RTM resistance corresponds to an interference with these systemic movement-promoting interactions. It is conceivable that the RTM system could prevent virus movement by sequestering the viral CP. An alternative possibility is that the same N-terminal region of $\mathrm{CP}$ could independently interact with movement-promoting host factors and with the RTM proteins. In this scenario, the same CP region would be related to virus restriction through its interaction with the RTM complex and, related to virus long-distance movement, through its interaction with other host factors. The RTM proteins and other host factors would thus compete for interaction with the $\mathrm{CP}$ sequence. It is worth noting that, while the PPV and LMV CP N-terminal regions are rather distinct (Fig. 3), both sequences are presumably able to interact with the same RTM complex. The fact that the RTM resistance is dominant is consistent with a direct or indirect interaction of the RTM proteins with viral movement proteins such as the $\mathrm{CP}$.

In both scenarios, if one of the RTM genes is mutated, as in the rtm mutants, the virus determinant would be free to interact with other host factors promoting movement. Similarly, amino acid modifications in the $\mathrm{CP} \mathrm{N}$-terminal region impeding the direct or indirect interaction of the capsid with the RTM complex would liberate the sequences involved in $\mathrm{CP}$-host factor interactions, thus promoting systemic movement. Of course, we cannot rule out the possibility that independent mechanisms of RTM restriction and support of virus movement coexist, involving distinct $\mathrm{CP}$ determinants and possibly other viral proteins and finely tuning the systemic invasion of the host plant.

\section{The sole CP primary sequence is not sufficient to explain the occurrence or not of RTM resistance.}

It is intriguing to note that all three LMV variant positions are located close to the DAG triplet in the N-terminal CP sequence (Fig. 3). The importance of this observation for the breaking of the RTM-mediated resistance is not yet clear. However, in PPV, the presence (in PPV-R) or the deletion (in PPV-NAT) of the 15 amino acids immediately downstream to the DAG (Fig. 3) does not seem to affect resistance-breaking ability, since both PPV isolates are able to mount a systemic infection in Col-0. In addition, introducing this 15-amino acid deletion in the $\mathrm{N}$-terminal $\mathrm{CP}$ region of pICPPVPSes did not alleviate its RTM-mediated restriction (data not shown). An- other intriguing remark is that the $\mathrm{N}$-terminal region upstream of the core conserved domain of the $\mathrm{CP}$ is significantly different between RTM-restricted PPV, LMV, and TEV isolates, both in length and in amino acid sequence (Fig. 3). However, all three viral proteins are presumably able to interact with the RTM proteins and, in doing so, to determine the outcome of the potyvirus infection. In consequence, except by postulating a role for shared posttranslational modifications, it is rather difficult to relate the CP-RTM interaction and specific amino acid motifs.

Several lines of evidence have suggested posttranslational modifications in the $\mathrm{CP} \mathrm{N}$-terminal region of potyviruses, either in the amino acid content, net charge, or phosphorylation or glycosylation state, affecting the ability of a virus to spread in its host plant (Chen et al. 2005; Fernández- Fernández et al. 2002; Ivanov et al. 2003). We initially hypothesized that they could affect not only the compatible interactions needed for systemic movement but also interactions with the potentially competing RTM system. Indeed, the CP of two potyviruses, Potato virus A (Ivanov et al. 2001) and PPV (Fernández- Fernández et al. 2002), have been shown to be phosphorylated, whereas the PPV $\mathrm{CP}$ is also modified by $O$-linked $N$-acetylglucosamination, possibly counteracting phosphorylation on Ser $+\mathrm{Thr}$ residues (Lefebvre et al. 2003). $O$-GlcNAc modification of PPV CP takes place in Arabidopsis and reduction of $O$-linked $N$-OGT activity in the sec-2 mutants has been shown to partially reduce virus spread and accumulation (Chen et al. 2005). The hypothesis that CP $O$-GlcNAcylation contributes to either PPV movement, RTM restriction, or both would be consistent with conclusions obtained recently with pumpkin phloem proteins (Taoka et al. 2007), which showed that protein-protein interactions between non-cell autonomous proteins (NCAP) and NCAP protein 1 and their subsequent transport through plasmodesmata and phloem are dependent on $O$-glycosylation or phosphorylation posttranslational modifications. However, computer predictions did not show a significant difference of total Thr and Ser phosphorylation or glycosylation residues between RTM-breaking and RTM-restricted PPV isolates. Moreover, this parameter cannot account for the behavior of the LMV RTM-breaking variants since only one of them has a mutation affecting a Ser or Thr residue (Fig. 3). The other two mutated residues (G and $D)$ are neither phosphorylated nor glycosylated. An alternative explanation would be that $O$-glycosylation might be influenced by the residues surrounding the modified amino acids and could be disturbed by changes in these residues in the RTM-breaking PPV isolates and the LMV-AFVAR variants. However, infection of sec-2 and spy-1 mutants in a Col-0 background with RTMrestricted LMV and PPV isolates (LMV-AF199, PPVPSes, and PPV-EA) did not result in a breakdown of the RTM resistance, clearly demonstrating that $O$-GlcNAc modification is not required for RTM restriction of LMV and PPV systemic infection in Col-0 plants.

In the present study, we showed that the CP N-terminal region is the key viral factor that determines the outcome of the interaction of two potyviruses, PPV and LMV, in Arabidopsis Col-0 plants. However, we were not able to reveal a clear correlation between the $\mathrm{CP} \mathrm{N}$-terminal primary sequence or its biophysical characteristics and the ability to break down RTM-mediated resistance. Therefore, the challenge ahead will be to understand how the RTM host proteins and the viral CP interplay to restrict viral long-distance movement.

\section{MATERIALS AND METHODS}

\section{Plant material.}

All plants were grown under greenhouse conditions. Arabidopsis thaliana Col-0 and Ler seeds were initially obtained 
from the Nottingham Arabidopsis Stock Centre (accessions N1092 and N8581, respectively). Chemically induced rtm mutants (Mahajan et al. 1998; Whitham et al. 1999) were provided by J. Carrington, Oregon State University (Corvallis, OR, U.S.A.) and $O G T$ mutants in Col-0 (sec-2 and spy-1) (Hartweck et al. 2002), by N. Oszewski, University of Minnesota (Minneapolis).

\section{Virus isolates and plasmid construction.}

PPV isolates were maintained in $N$. benthamiana plants. LMV-AF199, described by Krause-Sakate and associates (2002), was propagated in the lettuce cv. Trocadéro.

Isolates from three PPV strains were used in this study, namely PPV-R and PPV-NAT, belonging to the D strain (Maiss et al. 1989; Riechmann et al. 1990), PPV-PSes and PPV-SK68, belonging to the M strain (Palkovics et al. 1993; Sáenz et al. 2001), and PPV-EA, which typifies the El-Amar strain (Wetzel et al. 1991).

Construction of the PS/R and R/PS recombinant cDNA clones (Fig. 2) was described previously (Sáenz et al. 2000). Construction of pICPPVnk and pICPPVnk-GFP containing the full-length nucleotide sequence of PPV-R coupled or not with the GFP gene has also been described (Fernández-Fernández et al. 2001). pICPPVPSes (Salvador 2007), P/R(GFP) 7677 8576 and $\mathrm{P} / \mathrm{R}(\mathrm{GFP})$ 7677-9020, derive from the same pGPPVPS clone with A1395G and G1764A substitutions in the HCPro coding sequence (pGPPVPSes) that has been previously described (Sáenz et al. 2001). P/R(GFP) 7677-8576 and $\mathrm{P} / \mathrm{R}(\mathrm{GFP}) 7677-9020$ contain the GFP reporter gene and the $3^{\prime}$ NIb coding sequence $[\mathrm{P} / \mathrm{R}(\mathrm{GFP}) 7677-8576]$ or the $3^{\prime} \mathrm{NIb}$ and 5' CP coding sequence [P/R(GFP) 7677-9020] of pICPPVnkGFP replacing the original PPV-PS sequence of pICPPVPSes and pGPPVPSes, respectively (B. Salvador, P. Sáenz, J. B. Quiot, C. Simón-Mateo and J. A. García, unpublished results). In order to refine the position of the viral RTM-breaking determinant, new chimeric viruses were constructed as follows.

$R / P$ 8576-9020 and R/EA 8576-9020. pICPPVnk harbors a NaeI-KpnI site between the NIb and CP sequences (Fernández-Fernández et al. 2001), while all PPV isolates, PPV-R, PPV-PS, PPV-SK68, and PPV-EA included, present a unique $\operatorname{SacI}$ site about 455 bp downstream of the Ala (A) codon starting the $\mathrm{CP}$ coding sequence.

The PPV-PS 5' CP KpnI/SacI fragment was ligated into the $K p n I$ and $S a c I$ digested and linearized pICPPVnk vector. From PPV-EA, the 5' CP gene fragment was amplified by PCR with the following primers: CP PPV-EA/KpnI sense (GATGGTACC AATGTAGTTGTCCAT) and CP PPV-EA/SacI antisense (CT GTGGAGCTCGCGTGTTCGAC). KpnI and SacI restriction sites are underlined in the sense and antisense primers, respectively. The PPV-EA 5' CP gene PCR fragment was doubledigested with KpnI and $S a c \mathrm{I}$ and was substituted for the KpnISacI PPV-R fragment into the full-length pICPPVnk cDNA infectious clone. The final viral clones were then referred to as R/P 8576-9020 and R/EA 8576-9020, respectively.

$P / R$ 8576-9020 and P/SK68 8576-9020. The P/R 8576-9020 and P/SK68 8576-9020 recombinants were engineered by PCR in the pICPPVPSes cDNA infectious clone (Salvador 2007). Beforehand, the strategy implied the creation, into the pICPPVPSes clone, of a KpnI restriction site between the NIb and $\mathrm{CP}$ sequences, similarly to the pICPPVnk construct. For this purpose, we amplified first $1.2 \mathrm{~kb}$ of the NIb $3^{\prime}$ terminal region from pICPPVPSes with the following primers: $\mathrm{NIb}$ \#111)PPV-PSes sense (AAGGTTTTGAAGAATG) and NIb(\#940)PPV-PSes/KpnI antisense (GGTACCAGCCTGGTG TATAACAATGTTGG). The $K p n I$ restriction site is underlined. The PPV-PSes sequence 487 bp downstream of the hybridization site of the NIb(\#111)PPV-PSes sense primer displays a
SalI restriction site. The PCR fragment was consequently double digested with SalI and KpnI and was subcloned in a pCRIITOPO vector (Invitrogen, Carlsbad, CA, U.S.A.) upstream of a KpnI-SacI PPV-R or PPV-SK68 5' terminal CP gene fragment. The PPV-R KpnI-SacI 5' CP gene region was obtained by double KpnI and SacI digestion of the pICPPVnk clone and was subcloned in the recipient pCRII-TOPO vector. The PPVSK68 KpnI-SacI 5' CP gene region was amplified by PCR with the CP PPV-SK68/KpnI sense (CAGGCTGGTACCGAG GAAGACGAT) and CP PPV-SK68/SacI antisense (TTGTGG AGCTCGTGTGTTTGACAAG) primers. The fusion fragments obtained between the PPV-PSes $3^{\prime}$ terminal NIb gene region and the PPV-R or PPV-SK68 $5^{\prime}$ terminal CP gene region were substituted by triple digestion and ligation of SalI$B g l \mathrm{I}, B g l \mathrm{I}-S a c \mathrm{I}$, and SalI-SacI back into the full-length pICPPVPSes cDNA infectious clone. Clones were then referred to as P/R 8576-9020 and P/SK68 8576-9020, respectively.

\section{Virus inoculation methods.}

Arabidopsis rosette leaves were inoculated mechanically with extracts of $N$. benthamiana infected with the various PPV isolates as described by Decroocq and associates (2006). LMV inoculation of Arabidopsis plants was as described by Revers and associates (2003).

Transcripts were obtained for the five PS/R or R/PS recombinant constructs as well as for P/R(GFP) 7677-9020 using T7 RNA polymerase. They were then mechanically inoculated to $N$. benthamiana before transfer to Arabidopsis plants.

Because the second set of PPV recombinant isolates was constructed under the Cauliflower mosaic virus $35 \mathrm{~S}$ promoter, the infectivity of the newly obtained PPV constructs was assessed first on $N$. benthamiana and, later, directly on Arabidopsis Ler, Col-0, and rtm1, rtm2, and rtm3 plants by biolistic inoculation. Direct particle bombardment inoculation was also performed with the pICPPVnk, pICPPVPSes, PPVSK68, pICPPVnk-GFP, P/R(GFP) 7677-8576 clones, using a handheld device (Bio-Rad, Hercules, CA, U.S.A.) and starting from $0.1 \mu \mathrm{g}$ of infectious DNA per shooting.

To control the identity of the recombinant PPV clones, RTPCR was used to reamplify and sequence short fragments spanning the recombination points as detailed in Supplementary Table S1.

\section{Detection methods.}

Accumulation and spread of viral progenies originating from inoculating pICPPVnk-GFP, P/R(GFP) 7677-8576, and $\mathrm{P} / \mathrm{R}$ (GFP) 7677-9020 clones in the inoculated and noninoculated tissues of Arabidopsis plants was analyzed by monitoring of GFP fluorescence following the procedure of Decroocq and associates (2006).

Virus accumulation in uninoculated inflorescence tissues was assayed at $21 \mathrm{dpi}$ by double-antibody sandwich-ELISA using anti-PPV $\mathrm{CP}$ antibodies $(\mathrm{M}+\mathrm{D}$ antibody, LCA Laboratory, Blanquefort, France) and, if needed, confirming by RTPCR. Viral RNA was extracted and subjected to RT-PCR as described previously by Decroocq and associates (2006). LMV detection in Arabidopsis inflorescence tissues was carried out using ELISA as described by Revers and associates (2003).

\section{Sequencing of the LMV variant genome.}

Total RNA was extracted from LMV-infected Col-0 plants using the SV total RNA isolation system (Promega, Madison, WI, U.S.A.). First-strand cDNA synthesis was performed using the Superscript II reverse transcriptase using the LD-polyT primer (CACTGGCGGCCGCTCGAGCATGTACT ${ }_{30} \mathrm{NN}$ ). Five overlapping PCR fragments spanning the complete LMV genome were obtained using the Advantage 2 PCR kit (Clontech, 
Mountain View, CA, U.S.A.) and the following primer pairs: $0.1 \mathrm{cP}$ (AAAATAAAACAACCCAACACAACTC) and $0.2144 \mathrm{M}$ (CATTTGGATTGCGTCTTAGGTG); 0.1870P (GGCACAAG CATCGAACCATGT) and 0.4415M (G(G/A)CGAGTTGGTT CGATGAGT); 0.4148P (GAACTTTACTCTTCTCGCGTG) and 0.6702AM (TTTGG(G/A)ATCAGTGC(A/T)GGAGC); $0.5793 \mathrm{AP} \quad$ (TCTC(G/A)AACTTCTCCATTAGTAG) and 0.8072M (CAGCAAGCAGAGTGTCGAG); 0.7852P (GGCT GTTGGTGCACTATATAG) and LD-Prim (CACTGGCGGCC GCTCGAGCATGTAC). Cycling parameters were $1 \mathrm{~min}$ at $95^{\circ} \mathrm{C}$ followed by 30 cycles $\left(1 \mathrm{~min}\right.$ at $95^{\circ} \mathrm{C}, 3 \mathrm{~min}$ at $\left.68^{\circ} \mathrm{C}\right)$ and a final step of $3 \mathrm{~min}$ at $68^{\circ} \mathrm{C}$.

Automated DNA sequencing of the various uncloned PCR products was performed by Cogenics (Meylan, France). The sequence was compared with that of the published wild-type LMV-AF199 (GenBank AJ278854; Krause-Sakate et al. 2002) using ClustalW. Differences between the sequence of the variant and either the wild-type LMV-AF199 or other variants were confirmed by direct sequencing of viral cDNAs obtained as short PCR products spanning the regions of interest.

\section{Biophysical parameters of the $\mathrm{N}$-terminal $\mathrm{CP}$ region of PPV and LMV isolates.}

The predicted isoelectric point and the net charge were determined for the 90 or 39 first amino acids of the $\mathrm{CP}$ of various isolates or variants of PPV and LMV, respectively (Fig. 3), using the ExPASy ProtParam tool.

\section{ACKNOWLEDGMENTS}

We are grateful to L. Palkovics (Plant Pathology Department, Faculty of Horticultural Science, Corvinus University, Budapest, Hungary) for the PPVSK68 cDNA infectious clone. We thank T. Mauduit and M. Roncoroni for the production and maintenance of the Arabidopsis plants and J.-P. Eyquard for technical assistance. Experiments were carried out in compliance with the current French and European guidelines concerning quarantine and recombinant pathogens. This work was supported in part by the bilateral Hubert Curien France-Spain (PICASSO 11023ZA) and FranceSlovakia (STEFANIK 11130TM) funds. This work is currently funded by the European Union through the FP7 Small Collaborative Project KBBE204429 (SHARCO acronym). B. Salvador and J. A. García were also supported by grant BIO2007-67283 from Spanish Ministerio de Ciencia e Innovación. M. Glasa was supported by grant APVV-51-0402-07 from Slovak Research and Development Agency.

\section{LITERATURE CITATIONS}

Andersen, K., and Johansen, I. 1998. A single conserved amino acid in the coat protein gene of pea seed-borne mosaic potyvirus modulates the ability of the virus to move systemically in Chenopodium quinoa. Virology 241:304-311.

Atreya, C., Raccah, B., and Pirone, T. 1990. A point mutation in the coat protein abolishes aphid transmissibility of a potyvirus. Virology 178:161-165.

Blanc, S., López-Moya, J. J., Wang, R., Garcia Lampasona, S., Thornbury, D., and Pirone, T. 1997. A specific interaction between coat protein and helper component correlates with aphid transmission of a potyvirus. Virology 231:141-147.

Chen, D., Juárez, S., Hartweck, L., Alamillo, J. M., Simón-Mateo, C., Pérez, J. J., Fernández-Fernández, M. R., Olszewski, N. E., and García, J. A. 2005. Identification of Secret Agent as the $O$-GlcNAc transferase that participates in Plum Pox Virus infection. J. Virol. 79:9381-9387.

Chisholm, S. T., Mahajan, S. K., Whitham, S. A., Yamamoto, M. L., and Carrington, J. C. 2000. Cloning of the Arabidopsis RTM1 gene, which controls restriction of long-distance movement of tobacco etch virus. Proc. Nat. Acad. Sci. U.S.A. 97:489-494.

Chisholm, S. T., Parra, M. A., Anderberg, R. J., and Carrington, J. C. 2001. Arabidopsis RTM1 and RTM2 genes function in phloem to restrict longdistance movement of tobacco etch virus. Plant Physiol. 127:16671675.

Decroocq, V., Sicard, O., Alamillo, J.-M., Lansac, M., Eyquard, J.-P., García, J.-A., Candresse, T., Le Gall, O., and Revers, F. 2006. Multiple resistance traits control PPV infection in Arabidopsis thaliana. Mol.
Plant-Microbe Interact. 19:541-549.

Dolja, V. V., Haldeman-Cahill, R., Montgomery, A. E., Vandenbosch, K. A., and Carrington, J. C. 1995. Capsid protein determinants involved in cell-to-cell and long distance movement of Tobacco etch potyvirus. Virology 206:1007-1016.

Fernández-Fernández, M. R., Mouriño, M., Rivera, J., Rodríguez, F. Plana-Durán, J., and García, J. A. 2001. Protection of rabbits against rabbit hemorrhagic disease virus by immunization with the VP60 protein expressed in plants with a potyvirus-based vector. Virology 280:283-291.

Fernández- Fernández, M. R., Camafeita, E., Bonay, P., Méndez, E., Albar, J. P., and García, J. A. 2002. The capsid protein of a plant singlestranded RNA virus is modified by $O$-linked $N$-acetylglucosamine. J. Biol. Chem. 277:135-140.

Gilbertson, R. L., and Lucas, W. J. 1996. How do viruses traffic on the vascular highway? Trends Plant Sci. 1:260-268.

Hartweck, L. M., Scott, C. L. and Olszewski, N. E. 2002. Two $O$-Linked $\mathrm{N}$-Acetylglucosamine transferase genes of Arabidopsis thaliana L. Heynh. have overlapping functions necessary for gamete and seed development. Genetics 161:1279-1291.

Ivanov, K. I., Puustinen, P., Merits, A., Saarma, M., and Mäkinen, K. 2001. Phosphorylation down-regulates the RNA binding function of the coat protein of Potato Virus A. J. Biol. Chem. 276:13530-13540.

Ivanov, K., Puustinen, P., Gabrenaite, R., Vihinen, H., Rönnstrand, L., Vamu, L., Kalkkinen, N., and Mäkinen, K. 2003. Phoshorylation of the potyvirus capsid protein by protein kinase CK2 and its relevance for virus infection. J. Biol. Chem. 276:13530-13540.

Kehr, J., and Buhtz, A. 2008. Long distance transport and movement of RNA through the phloem. J. Exp. Bot. 59:85-92.

Kimalov, B., Gal-On, A., Stav, R., Belausov, E., and Arazi, T. 2004. Maintenance of coat protein $\mathrm{N}$-terminal net charge and not primary sequence is essential for zucchini yellow mosaic virus systemic infectivity. J. Gen. Virol. 85:3421-3430.

Krause-Sakate, R., Le Gall, O., Fakhfakh, H., Peypelut, M., Marrakchi, M., Varveri, C., Pavan, M. A., Souche, S., Lot, H., Zerbini, F. M., and Candresse, T. 2002. Molecular characterization of Lettuce mosaic virus field isolates reveals a distinct and widespread type of resistance-breaking isolate : LMV-Most. Phytopathology 92:563-572.

Lefebvre, T., Ferreira, S., Dupont-Wallois, L., Bussiere, T., Dupire, M. J., Delacourte, A., Michalski, J. C., and Caillet-Boudin, M. L. 2003. Evidence of a balance between phosphorylation and O-GlcNAc glycosylation of Tau proteins - A role in nuclear localization. Biochim. Biophys. Acta 1619:167-176.

López-Moya, J., and Pirone, T. 1998. Charge changes near the $\mathrm{N}$ terminus of the coat protein of two potyviruses affect virus movement. J. Gen. Virol. 79:161-165.

Lough, T. J., and Lucas, W. J. 2006. Integrative plant biology: Role of phloem long-distance macromolecular trafficking. Ann. Rev. Plant Biol. 57:203-232.

Lucas, W. J. 2006. Plant viral movement proteins: Agents for cell-to-cell trafficking of viral genomes. Virology 344:169-184.

Mahajan, S. K., Chisholm, S. T., Whitham, S. A., and Carrington, J. C. 1998. Identification and characterization of a locus (RTM1) that restricts long-distance movement of tobacco etch virus in Arabidopsis thaliana. Plant J. 14:177-186.

Maiss, E., Timpe, U., Brisske, A., Jelkmann, W., Casper, R., Himmler, G., Mattanovich, D., and Katinger, H. W. D. 1989. The complete nucleotide sequence of Plum pox virus RNA. J. Gen. Virol. 70:513-524.

Nicaise, V., Gallois, J.-L., Chafiai, F., Allen, L. M., Schurdi-Levraud, V., Browning, K. S., Candresse, T., Caranta, C., Le Gall, O., and GermanRetana, S. 2007. Coordinated and selective recruitment of eIF4E and eIF4G factors for potyvirus infection in Arabidopsis thaliana. FEBS (Fed. Eur. Biochem. Soc.) Letters 581:1041-1046.

Palkovics, L., Burgyán, J., and Balázs, E. 1993. Comparative sequence analysis of four complete primary structures of plum pox virus strains. Virus genes 7:339-347.

Rakitina, D. V., Kantidze, O. L., Leshchiner, A. D., Solovyev, A. G., Novikov, V. K., Morozov, S. Y., and Kalinina, N. O. 2005. Coat proteins of two filamentous plant viruses display NTPase activity in vitro. FEBS (Fed. Eur. Biochem. Soc.) Lett. 579:4955-4960.

Revers, F., Le Gall, O., Candresse, T., and Maule, A. J. 1999. New advances in understanding the molecular biology of plant/potyvirus interactions. Mol. Plant-Microbe Interact. 12:367-376.

Revers, F., Guiraud, T., Houvenaghel, M. C., Mauduit, T., Le Gall, O., and Candresse, T. 2003. Multiple resistance phenotypes to Lettuce mosaic virus among Arabidopsis thaliana accessions. Mol. Plant-Microbe Interact. 16:367-376.

Riechmann, J. L., Lain, S., and Garcia, J. A. 1990. Infectious in vitro transcripts from a Plum pox potyvirus cDNA clone. Virology 177:710-716.

Rojas, M. R., Zerbini, F. M., Allison, R. F., Gilbertson, R. L., and Lucas, 
W. J. 1997. Capsid protein and helper component-proteinase function as potyvirus cell-to-cell movement proteins. Virology 237:283-295.

Sáenz, P., Cervera, M. T., Dallot, S., Quiot, L., Quiot, J. B., Riechmann, J. L., and García, J. A. 2000. Identification of a pathogenicity determinant of Plum pox virus in the sequence encoding the C-terminal region of protein P3+6K1. J. Gen. Virol. 81:557-566.

Sáenz, P., Quiot, L., Quiot, J. B., Candresse, T., and García, J. A. 2001. Pathogenicity determinants in the complex virus population of a Plum pox virus isolate. Mol. Plant-Microbe Interact. 14:278-287.

Salvador, B. 2007. Pathogenicity and host range determinants in Plum pox virus infection: A role for P1 protein, Universidad Autónoma de Madrid (Spain).

Shukla, D. D., and Ward, C. W. 1989. Structure of potyvirus coat protein and its application in the taxonomy of the potyvirus group. Adv. Virus Res. 36:273-314.

Taoka, K.-I., Ham, B.-K., Xoconostle-Cázares, B., Rojas, M. R., and Lucas, W. J. 2007. Reciprocal phosphorylation and glycosylation recognition motifs control NCAPP1 interaction with pumpkin phloem proteins and their cell-to-cell movement. Plant Cell 19:1866-1884.

Urcuqui-Inchima, S., Haenni, A.-L., and Bernardi, F. 2001. Potyvirus proteins: A wealth of functions. Virus Res. 74:157-175.

Waigmann, E., Ueki, S., Trutnyeva, K., and Citovsky, V. 2004. The ins and outs of nondestructive cell-to-cell and systemic movement of plant viruses. Crit. Rev. Plant Sci. 23:195-250.

Wang, H. L., Sudarshana, M. R., Gilbertson, R. L., and Lucas, W. J. 1999. Analysis of cell-to-cell and long distance movement of a Bean dwarf mosaic geminivirus-Green fluoresecnt protein reporter in host and non host species: Identification of sites of resistance. Mol. Plant-Microbe Interact. 12:345-355

Wetzel, T., Candresse, T., Ravelonandro, M., Delbos, R. P., Mazyad, H., Aboul-Ata, A. E., and Dunez, J. 1991. Nucleotide sequence of the 3'terminal region of the RNA El Amar strain of Plum pox potyvirus. J. Gen. Virology 72:1741-1746.

Whitham, S. A., Yamamoto, M. L., and Carrington, J. C. 1999. Selectable viruses and altered susceptibility mutants in Arabidopsis thaliana. Proc. Nat. Acad. Sci. U.S.A. 96:772-777.

Whitham, S. A., Anderberg, R. J., Chisholm, S. T., and Carrington, J. C. 2000. Arabidopsis RTM2 gene is necessary for specific restriction of tobacco etch virus and encodes an unusual small heat shock-like protein. Plant Cell 12:569-582.

AUTHOR-RECOMMENDED INTERNET RESOURCE

ExPASy ProtParam tool: au.expasy.org/tools/protparam.html 